\title{
Self-assessment questions: Recent advances in the risk assessment and treatment of osteoporosis
}

\author{
Juliet Compston
}

1 The following interventions have been shown to reduce hip fracture in postmenopausal women:
(a) Zoledronic acid
(b) Denosumab
(c) Alendronate
(d) Ibandronate
(e) Strontium ranelate

2 The following risk factors act independently of bone mineral density (BMD) to increase the risk of fracture:
(a) Glucocorticoids
(b) Aromatase inhibitor therapy
(c) Falls
(d) Premature menopause
(e) Family history of hip fracture

3 The following statements are true:

(a) Obesity is protective against fractures

(b) The effect of glucocorticoids on fracture risk is dose dependent

(c) The risk of atypical femoral fractures increases with duration of bisphosphonate therapy

(d) Most postmenopausal women with fracture have osteoporosis (defined as BMD T-score $\leqslant-2.5$ )

(e) The risk of osteonecrosis of the jaw is increased in patients receiving denosumab therapy

Answers to these self-assessment questions can be found on page 592 . 